\title{
Bricolages en design
}

Inventer des rapports non réguliers à la technique

\section{Sophie Fétro}

\section{(2) OpenEdition}

Journals

Édition électronique

URL : https://journals.openedition.org/tc/7577

DOI : $10.4000 /$ tc. 7577

ISBN : 0248-6016

ISSN : 1952-420X

Éditeur

Éditions de l'EHESS

\section{Édition imprimée}

Date de publication : 24 décembre 2015

Pagination : 152-167

ISBN : 978-2-7132-2505-5

ISSN : 0248-6016

\section{Référence électronique}

Sophie Fétro, « Bricolages en design », Techniques \& Culture [En ligne], 64 | 2015, mis en ligne le 24 décembre 2018, consulté le 29 septembre 2022. URL : http://journals.openedition.org/tc/7577 ; DOI : https://doi.org/10.4000/tc.7577 


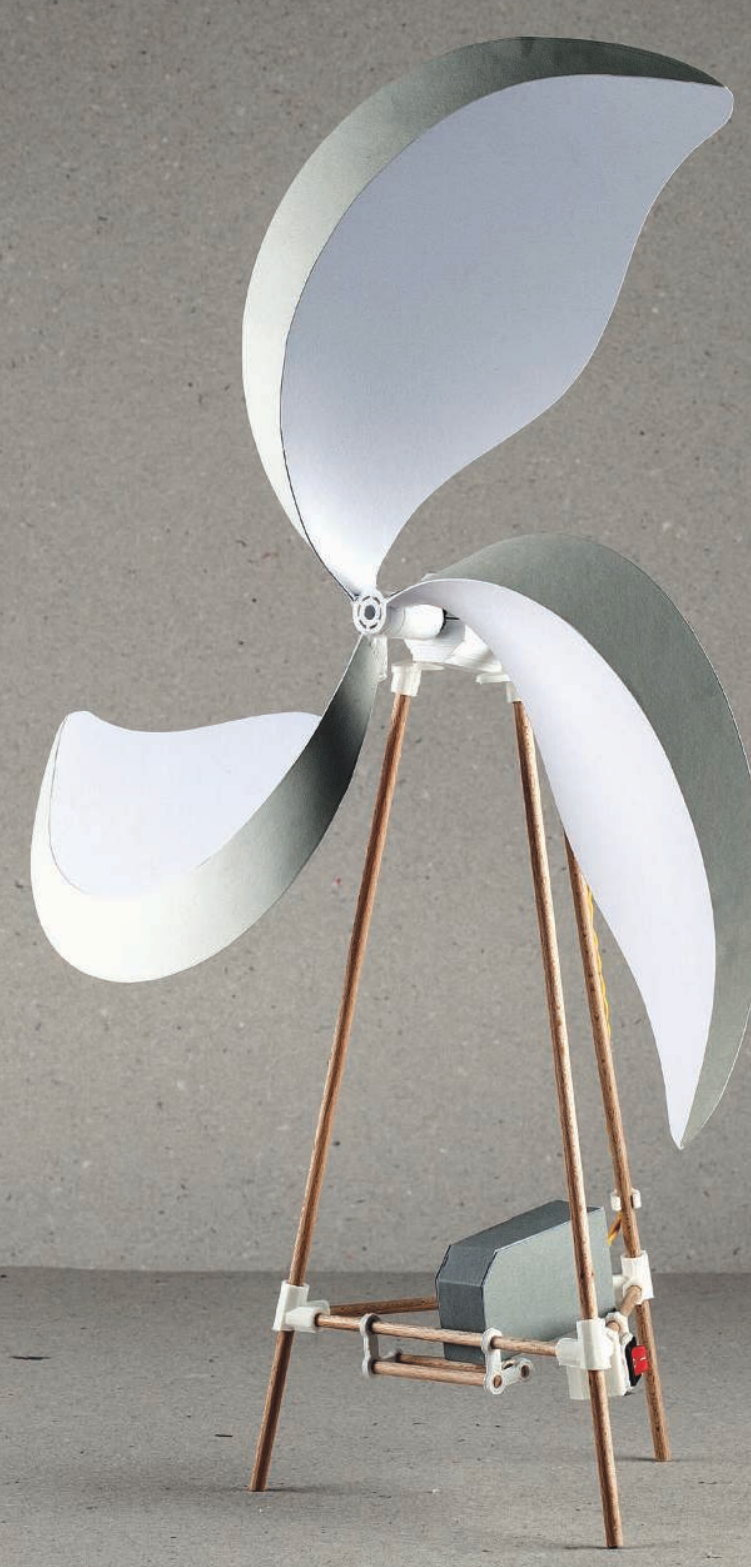




\section{Bricolages en design}

\section{Inventer des rapports non réguliers à la technique}

Le rapport du design au bricolage ne va pas de soi, d'abord parce que le design est associé avant tout à l'industrie, à la production de masse en série, ensuite parce que ces deux univers semblent de prime abord éloignés l'un de l'autre. Pourtant ce rapprochement semble se préciser avec le développement du numérique, de l'open data, de l'impression 3D et des FabLabs. Il s'agit alors d'en repérer les différentes manifestations, de débusquer les a priori qui pourraient concerner cette rencontre, d'identifier les rapports de force et tensions sous-jacents de cette convergence à partir d'une étude à la fois sémantique et critique en adoptant une méthodologie réflexive et comparative spécifique à la théorie du design et à l'esthétique. Cette rencontre du design et du bricolage n'est pas si récente, et il serait erroné de penser que le numérique l'occasionne, néanmoins on peut penser que ce dernier actualise des formes plus anciennes de bricolage (ou les rend plus manifestes encore qu'elles ne l'étaient auparavant) tandis qu'il en inaugure d'autres.

Tout d'abord, rappelons que le bricolage n'a pas toujours été l'activité domestique de semi-loisir (Coulangeon, Menger \& Roharik 2002: 39-55) auquel le mot est aujourd'hui couramment associé. Emprunté à l'italien briccola (Battaglia 1962: 372) qui désigne originellement une sorte de catapulte, le terme «bricolage » dérive de «brigole (Imbs 1975: 950), qui donnera par la suite « bricole», et du radical brikkil « celui qui brise » (Rey 2005: 173). « Bricole», engin « destiné[e] à démolir les murailles » (Littré 2000), provient par ailleurs de l'allemand brëchel dont découle le verbe brechen signifiant « rompre, casser » (Imbs 1975). En ce sens, et contre toute attente, le bricolage concerne des dispositifs offensifs, à vocation essentiellement militaire, destinés à détruire quelque chose. C'est donc en tant que «machine de guerre » (Dupré 1972: 325) que la « bricole» peut être historiquement envisagée. Dans ce contexte, l'intelligence technique qui est convoquée relève avant tout de la stratégie militaire conduisant à la fabrication d'engins spécifiques: trébuchets, catapultes, onagres, mangonneaux. Depuis le Moyen Âge, plusieurs recueils illustrés attestent de la création de dispositifs guerriers permettant d'organiser le siège 
des villes ou leur défense. Qu'il s'agisse des dessins réalisés par Villard de Honnecourt (Carnet, 1220-1250), d'enluminures du Moyen Âge (comme celles présentes dans les Fleurs des chroniques de Bernard Gui, 1365-1370), des figurations de Mariano di Jacopo Taccola (Liber tertius de ingeneis ac edifitiis non usitatis, 1433), des planches du Codex Atlanticus de Léonard de Vinci (1487), des planches de l'Encyclopédie de Diderot et d'Alembert (1751-1772) ou des illustrations d'Eugène Viollet-le-Duc (Dictionnaire raisonné de l'architecture française du XIe au XVI siècle, 18541868, tome 5), les représentations de catapultes sont nombreuses, attestant non seulement de connaissances techniques et scientifiques savantes (à propos notamment de la chute des corps pesants) mais également d'un sens esthétique et d'un plaisir à rendre visible et compréhensible des mécanismes plus ou moins complexes. Fait remarquable, au Moyen Âge, les ingénieurs militaires sont parfois médecins et anatomistes (Guido da Vigevano) ou bien hommes de lettres, peintres et/ou architectes. Bien que l'on puisse trouver des ouvrages spécifiques dédiés à l'art de la guerre, il n'y a pas toujours de distinction franche entre des machines à vocation militaire et celles destinées à des applications civiles, agricoles ou architecturales comme en témoigne le recueil de Villard de Honnecourt dans lequel se mêlent sur une même planche une scie mécanique, un piège à arbalète, un mécanisme d'ange mécanique, un appareil élévatoire à vis ainsi qu'un aigle mobile pour lutrin (Gimpel 2002: 121). Par conséquent, le bricolage est aussi bien l'expression d'une forme d'ingéniosité technique que d'un attrait pour les mèkhanai ${ }^{1}$.

\section{Bricolages offensifs}

Aujourd'hui, la communication en réseau, le partage des données et le développement d'outils de type imprimantes 3D (notamment les RepRap ${ }^{2}$ ), pourraient ressusciter cette origine du mot « bricolage » en tant qu'activité productrice de dispositifs techniques offensifs, rendant ainsi possible l'existence (bien que pour le moment minoritaire) d'une industrie militaire de proximité quasiment indépendante dans sa mise en œuvre. La fabrication « maison » de drones, d'armes ${ }^{3}$, de dispositifs de surveillance, « bricolés » en toute autonomie par leurs usagers eux-mêmes, rappelle finalement très bien que le bricolage n'est pas toujours neutre et sans dommage pour les individus. Dans le cas d'un bricolage offensif, l'intelligence technique qui est impliquée, passe alors, pour reprendre la distinction que fait Michel de Certeau, de la tactique en tant qu'« art du faible » à la stratégie en tant que « calcul (ou manipulation) des rapports de force » (de Certeau 1990: 61). Par conséquent, le bricolage, ancré historiquement dans l'aprèsguerre comme moyen d'aide à la reconstruction et indissociable de la société de loisirs, pourrait bien s'avérer beaucoup plus ambigu qu'il n'y paraît, posant de fait des questions éthiques et de mours. Cette orientation du design vers un bricolage offensif peut constituer un premier repère $^{4}$, une limite, un point de rupture marquant un changement de direction quant à la finalité du dispositif technique mis au point, lieu de bascule, pourrait-on dire, entre un génie appliqué à des fins militaires et une ingéniosité appliquée à des fins pacifiques. 


\section{Une attitude qui consiste à aller " par-ci, par-là "}

Par ailleurs, il ne suffit pas que les dispositifs techniques soient fabriqués manuellement pour qu'il soit question de bricolage. Dans le champ du design, certaines démarches peuvent s'y rapporter alors même que les machines employées sont industrielles et que le travail manuel n'est que partiellement sollicité. C'est alors la façon de les employer qui induit le bricolage, et non la nature même de l'outil ou la sollicitation corporelle qu'il pourrait impliquer. Aussi, c'est à une autre définition de la notion de bricolage à laquelle nous pouvons nous référer à présent car le mot « bricolage » peut désigner une attitude qui consiste à « aller par-ci, par-là » (1480). « Bricoler » c'est, notamment au billard, l'action qui consiste à savoir utiliser le rebond de la balle contre les bords de la bande de la table, d'où « ricocher » en parlant d'une balle ou d'une bille. Le terme qualifie également une sorte de ruse, qui peut parfois viser à abuser d'autrui, à « manœuvrer sournoisement »; la formule « donner la bricole à quelqu'un » signifiant « tromper quelqu'un » en atteste. Le bricolage, comme toute mètis (Detienne \& Vernant 1974), est donc ambivalent à plus d'un titre. Toutefois, il est possible de concevoir le bricolage comme une attitude propice à la découverte et à la recherche. Bricoler pourrait alors coïncider avec une tournure d'esprit spécifique consistant à laisser vagabonder l'imagination par « mouvements incidents » (Lévi-Strauss 1962: 30), en procédant par petits sauts ${ }^{5}$. En cela, le bricolage ne désigne pas seulement un travail manuel fait de menus travaux, il constitue plutôt une méthodologie créative « engagée dans le devenir et l'action » (Detienne \& Vernant 1974) : une heuristique favorisant la découverte et l'exploration, une façon d'aller obliquement, impliquant à la fois des ruptures et de la coïncidence. Cette conduite pourrait caractériser l'approche du designer et la nature de son travail créatif dans sa dimension prospective. Bricoler correspondrait donc, dans le champ du design, à une « attitude » mentale spécifique (Moholy-Nagy 1947: 277) ne relevant en rien de la tromperie bien que procédant par ruse et ricochets, ce qui consiste à privilégier un rapport ouvert à la technique et pour lequel tout n'est pas prédéterminé. Faire du design serait donc par essence bricoler, autrement dit, mettre en œuvre les conditions propices à une approche en zigzag, non rectiligne, du projet. Toute la question repose alors désormais sur la possibilité de suivre une telle approche, favorable à l'imprévu, alors même que les technologies auxquelles les designers recourent sont régies par le calcul et des programmes. Dès lors, comment peuvent-ils continuer à bricoler avec des dispositifs techniques pour lesquels tout semble déjà décidé d'avance? Ainsi, l'emploi d'ordinateurs et de machines pilotées numériquement favorise-t-il ce vagabondage ou l'empêche-t-il ?

\section{S'émanciper des paramétrages d'usine}

Avec le numérique la panoplie du bricoleur augmente, et l'on peut espérer, comme le dit Bruno Latour, que de ce fait « son habileté s'accroît » (2010). Si cette idée peut être nuancée au regard de la quantité de gadgets générés par impression 3D, l'accès à un outillage de pointe (ordinateur, 
commandes numériques, scanners 3D, programmes open source, etc.) élargit le champ d'exploration et de recherche des designers. Si, malgré tout, l'habileté du bricoleur ne s'en trouve pas décuplée, ce sont néanmoins ses possibilités techniques qui s'étendent. Aussi, il devient désormais possible de parler de bricolage numérique à l'égard du design, dès lors qu'il est question pour les designers de « bidouiller » les lignes de code et d'intervenir sur les paramétrages des programmes. Le travail de Marc Fornes, chef de fil de l'agence THEVERYMANY, va dans le sens d'un bricolage sophistiqué et exigeant, qui intègre pourtant l'erreur, le «bug », comme donnée essentielle du travail de programmation. Il parle ainsi d'« indétermination précise » (Fornes 2012) :

«Toutes les structures que nous faisons sont précises, parce qu'elles sont déterminées sous formes de valeurs numériques. Si on exécute plusieurs fois le même code on obtient plusieurs fois la même structure, ce qui est important pour pouvoir corriger des moments. Mais il est quand même question d'“indétermination" parce que tous les codes qu'on écrit comportent tellement d'étapes qu'on est souvent obligé de les exécuter pour voir ce qui en résulte, pour les évaluer, les corriger, les exécuter à nouveau ».

Si jusqu'à présent, les designers s'étaient ouverts au numérique via l'utilisation de logiciels de traitement de l'image et de modélisation 3D, plusieurs se tournent également vers le paramétrage des outils de fabrication. Le travail de programmation, l'écriture de lignes de code, le détournement de programmes de pilotage des machines et la création de plugins (extensions de fonctionnalités d'un programme) constituent un travail spécifique leur permettant d'échapper à un déterminisme trop important induit par les logiques commerciales propriétaires. Ainsi, les «bricolages » auxquels s'adonnent les designers ne consistent pas à répéter des savoir-faire existants ; il s'agit au contraire pour eux de les interroger et de les déranger positivement. Aussi, les designers ne sont pas seulement usagers des technologies car ils les modifient, les transforment, les détournent de leurs applications premières, opérant une critique des usages officiellement établis. Les approches de plusieurs designers recourant au numérique nuancent le propos de Claude Lévi-Strauss lorsqu'il dit : « de nos jours, le bricoleur reste celui qui œuvre de ses mains, en utilisant des moyens détournés » (1962). En réalité, le bricolage numérique retient surtout le principe du détournement comme modalité opératoire. Parler de bricolage numérique n'est donc pas en cela contradictoire. Cette façon de procéder atteste que les technologies numériques peuvent se prêter au bricolage, favorisant ainsi un rapport exploratoire à la technique.

\section{Le bricolage comme positionnement critique}

Le bricolage en design peut également relever d'une approche critique à l'encontre de la société de consommation. En mettant à la disposition du public des plans ainsi qu'un matériel élémentaire permettant aux visiteurs de construire eux-mêmes différents objets domestiques: tables, chaises, bancs, étagères, etc. Proposta per un'autoprogettazione (1974, Galleria 
Milano), proposition manifeste d'Enzo Mari, est une incitation radicale à penser autrement les processus de production et de consommation, opérant une critique des logiques consuméristes capitalistes. Aujourd'hui, le bricolage peut encore être perçu comme l'expression d'une sorte de méfiance, notamment à l'encontre des technologies et des programmes tout faits, conduisant les designers à se tourner du côté du « Do It Yourself» (DIY). En développant un art spécifique du bricolage, fabricant par exemple leurs propres moyens de production, les designers tentent certainement de s'affranchir des systèmes qui s'imposent à eux. Vilém Flusser indique que : «bien des choses de mon environnement ne m'inspirent pas franchement confiance. Peut-être parce que j'ai l'air de me servir d'elles, alors qu'en réalité je sais que je les sers » (1996: 5). Malgré toutes les réticences qui peuvent s'exprimer à l'encontre de l'industrie et des technologies, il est important de relever que les bricolages atypiques que développent les designers ne génèrent pas seulement des objets fonctionnels peu fiables ou des «non-choses» (Flusser 1996). Les bricolages des designers ne correspondent ni véritablement à des dispositifs au sens où l'entend Giorgio Agamben en tant qu'ils ont « la capacité de capturer, d'orienter, de déterminer, d'intercepter, de modeler, de contrôler et d'assurer les gestes, les conduites, les opinions et les discours des êtres vivants » (Agamben 2007: 31), ni à des «trucs idiots » (Flusser 1996) ou à des « appareils» dont la complexité

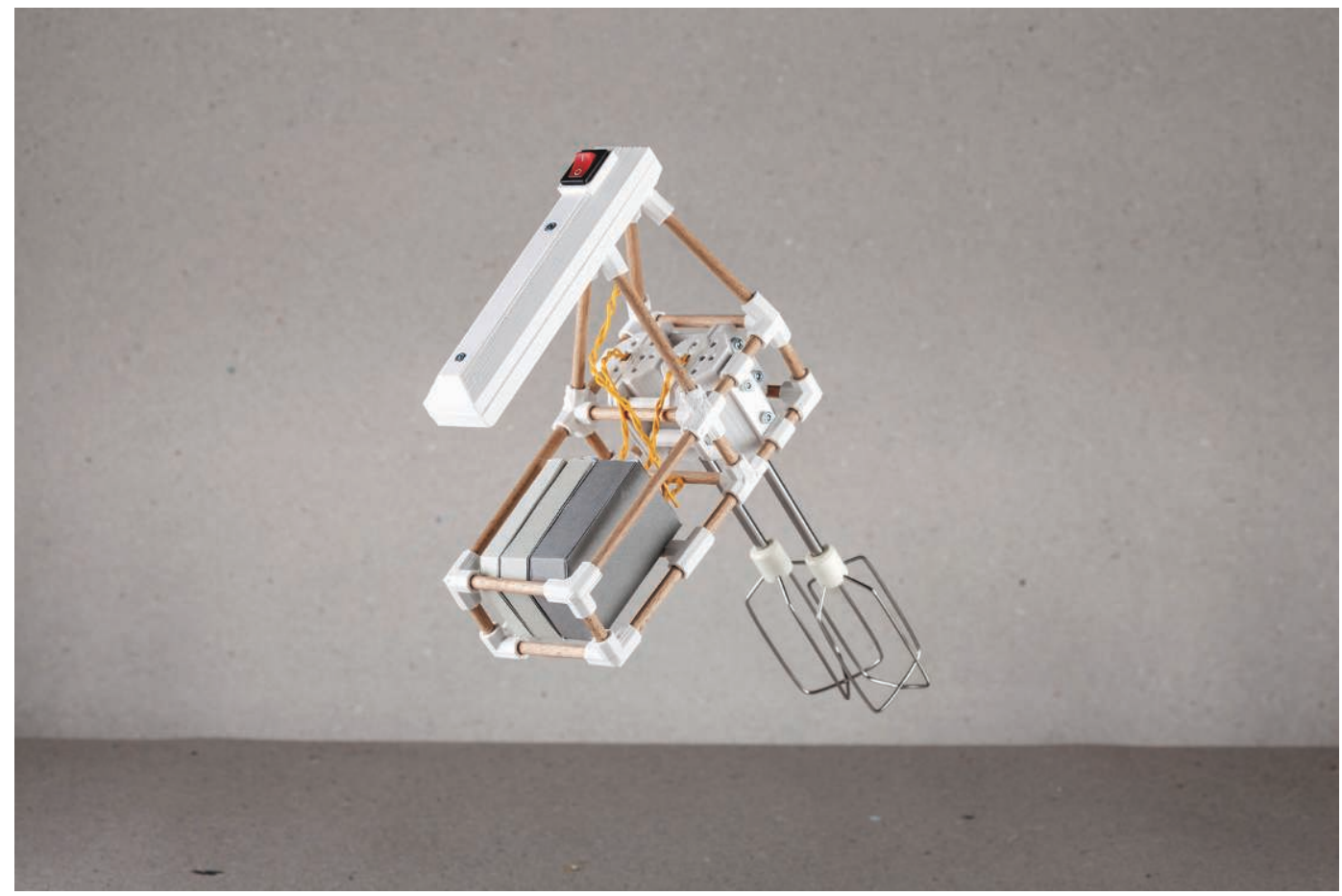

. Batteur électrique. Série Hacking Households, 2014. Design : Jesse Howard, Leonardo Amico, Thibault Brevet, Coralie Gourguechon, Jure Martinec, Nataša Muševič, and Tilen Sepič. 
interne, selon Vilém Flusser, ne permet pas d'en comprendre le fonctionnement. En effet, ils n'engendrent ni une « saisie» du corps et de l'esprit (Agamben 2007), ni un « conditionnement $»$ des usagers (Flusser 1996). Si les bricolages des designers pourraient, aux yeux de certains, s'apparenter à un retour en arrière, voire à de la technophobie, en réalité, ils ne rejettent en rien les dernières avancées technologiques. Les objets de Jesse Howard opèrent, par exemple, un mélange de technologies de pointe et de bricolages maison. S'appuyant à la fois sur la récupération d'objets existants, l'utilisation de composants standards et la fabrication de pièces spécifiques via la modélisation et l'impression 3D, il propose à tout un chacun, et quasiment n'importe où (pour peu qu'un FabLab se trouve à proximité), de réaliser des appareils électroménagers (batteur électrique, bouilloire électrique, grille-pain) qui peuvent être montés et démontés, modifiés et réparés à loisir. Pour cela, il met en ligne gratuitement les instructions nécessaires à leur fabrication et à leur montage ainsi que les liens permettant de télécharger librement les fichiers numériques correspondant via les sites de libre partage de données Thingiverse et OpenStructure.

Les propositions de Jesse Howard sont une invitation à la débrouille, à la récupération et à un emploi rusé des technologies d'impression 3D. Non seulement le designer bricole, mais il enjoint également l'usager à s'impliquer dans la production, la réparation et la modification de ses propres objets techniques. Il s'affranchit ainsi en partie des logiques commerciales et de la logique d'obsolescence programmée consistant à réduire la durée de vie d'un objet pour en accroître le renouvellement. Les bricolages de Jesse Howard constituent en cela une critique nécessaire et salvatrice à l'égard de la production de masse et des logiques productivistes et consuméristes héritées des XIXe et $\mathrm{xx}^{e}$ siècles. Loin de reproduire exactement d'anciens procédés, il combine savamment des techniques et principes productifs qui ont fait leurs preuves (résistance électrique, standardisation, production en série) aux possibilités offertes par les technologies numériques (production à la demande, partage des données, adaptabilité), prenant en considération des préoccupations contemporaines aussi bien économiques (sortir d'un gaspillage productif), sociales (s'émanciper d'une logique propriétaire pour faire place à une relation d'échange, de partage, de récupération, de transmission et de transit des objets) qu'environnementales (prendre en considération les méfaits d'une logique de production illimitée sur la biosphère et la santé des individus). Cette orientation du design du côté du bricolage va dans le sens d'une meilleure connaissance des objets techniques et démontre qu'il est possible de faire exister un rapport alternatif aux usages dominants qui s'imposent aujourd'hui de façon globalisée. 


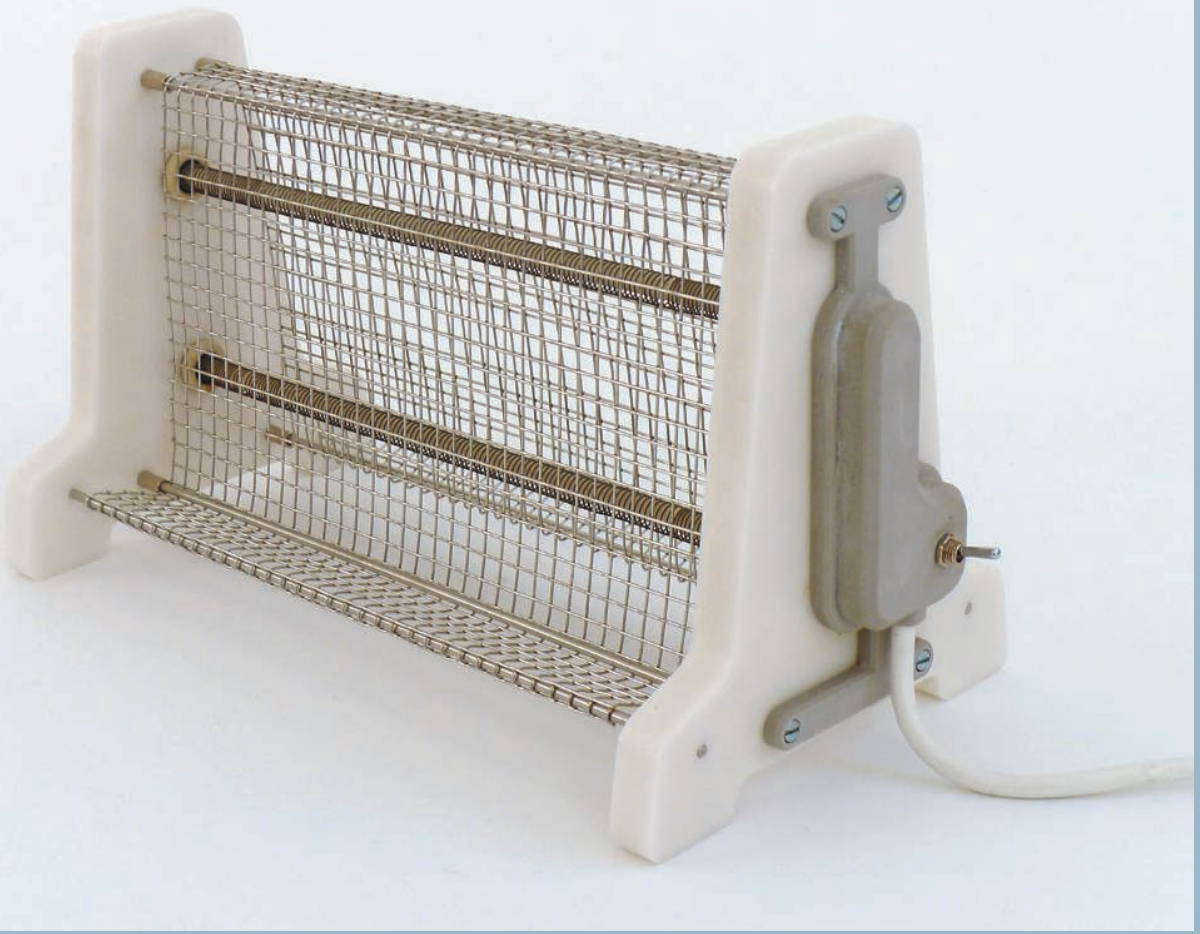

Grille-pain. Toaster, 2012. Design : Jesse Howard.

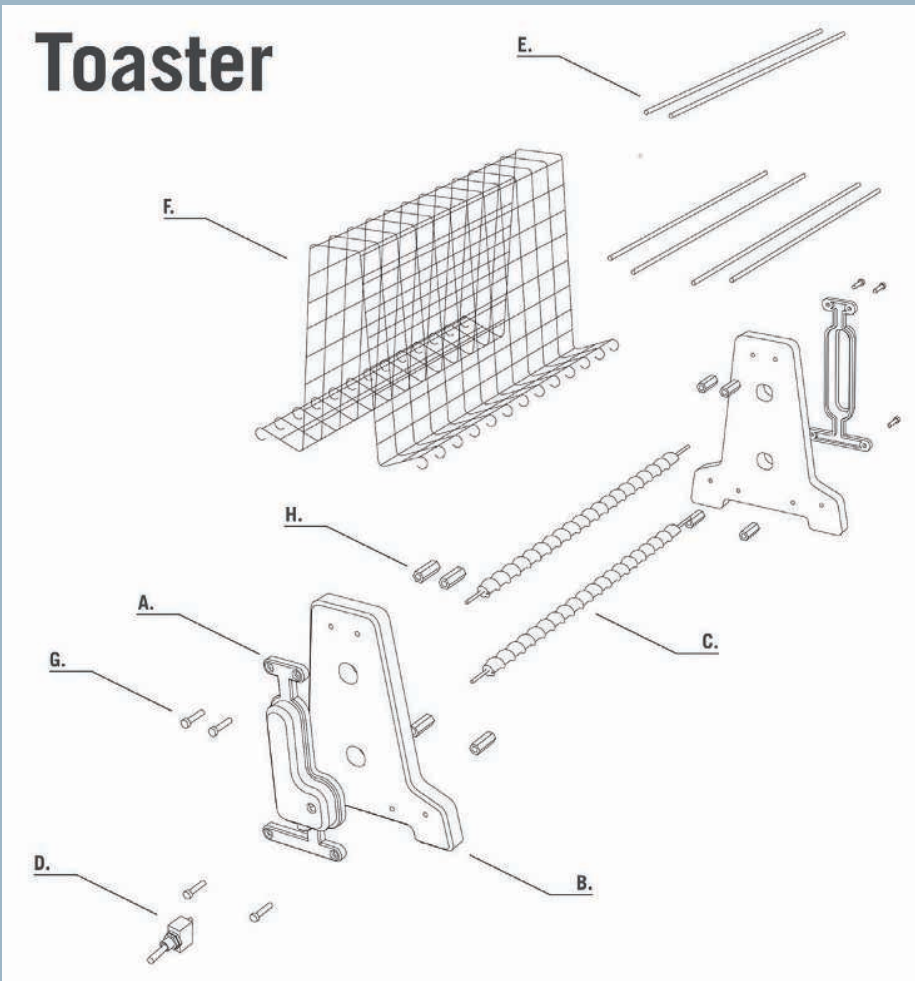

A. Switch Covers - 3D Printed OpenStructures Compatible
thingiverse.com/thing:25803

B. Side Panels - CNC Milled

OpenStructures Compatible

c. Heating Elements

Recouperated

from Tefal Express LS or Similar

D. Toggle Switch

Standard Component

rs-online.com / item\# 734-7141

E. Stainless Steel Mesh (1 mm) Standard Component

F. M3 Stainless Steel Bars Standard Component

\section{G. $20 \mathrm{~mm}$ M3 Bolts} Standard Component

H. $10 \mathrm{~mm}$ M3 Standoffs Standard Component

Mode d'emploi. Grille-pain, Transparent Tools: Manuals, 2012. Design : Jesse Howard. 


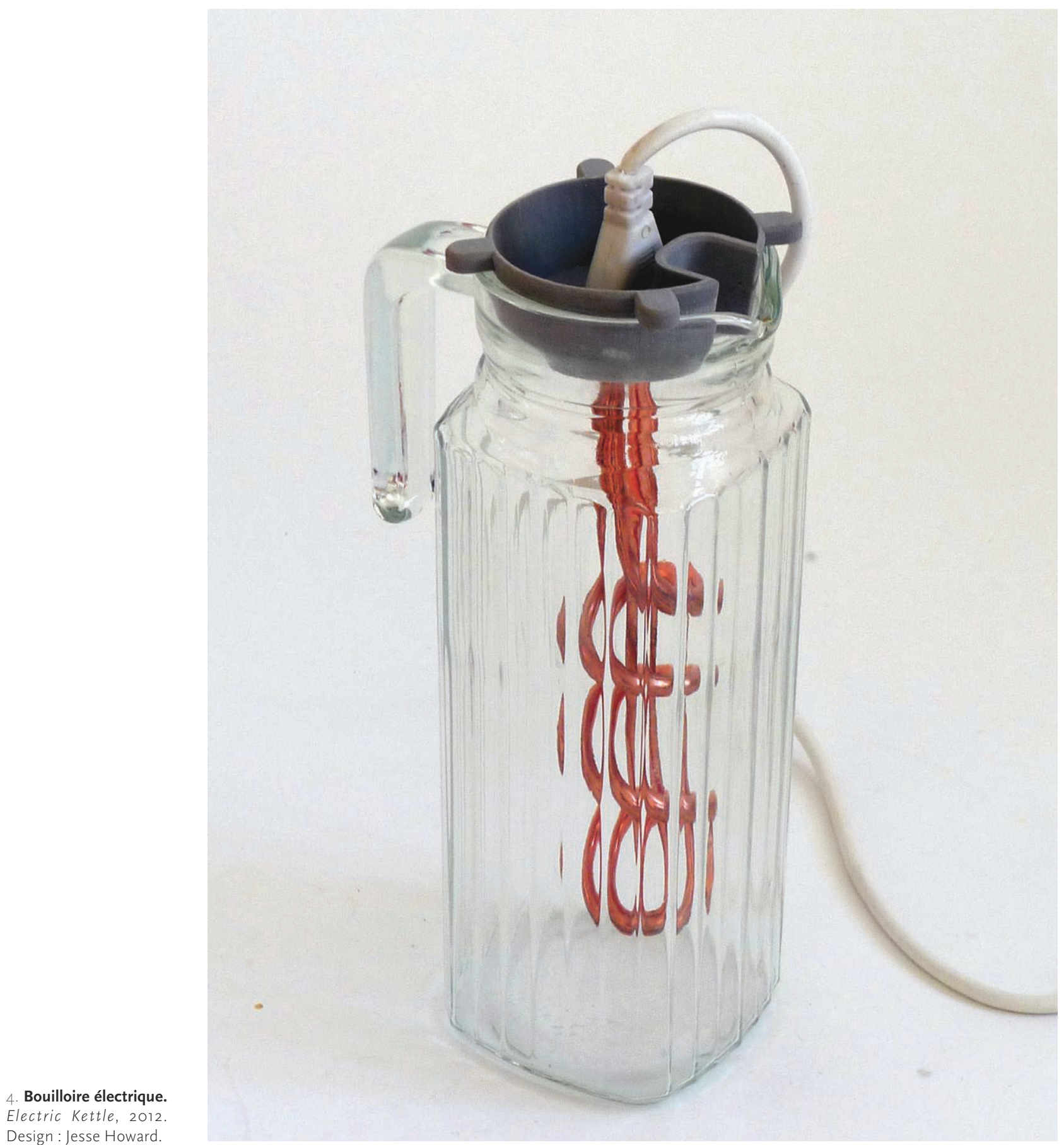




\section{Electric Kettle}

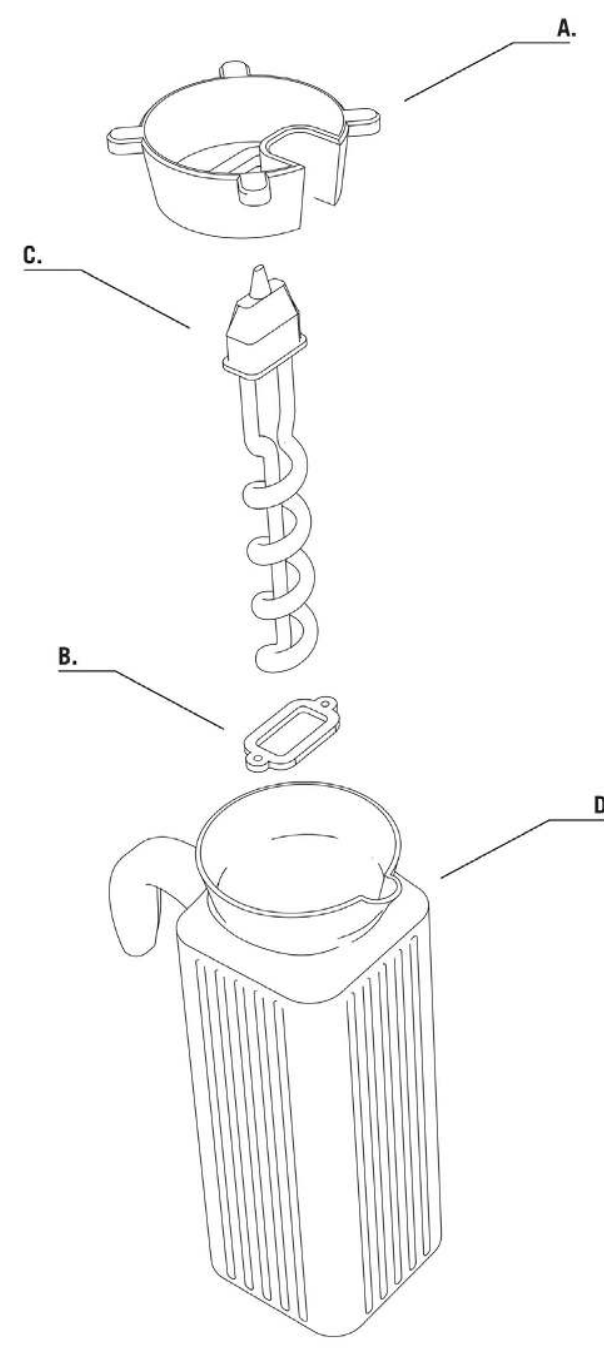

A. Lid

3D Printed

www.thingiverse.com/thing:25795

B. Adaptor - Laser Cut Plastic

OpenStructures Compatible

www.thingiverse.com/thing:25796

C. Heating Element

220v 1500w

ebay: "immersion water heater"

D. Glass Pitcher

Standard component

Mode d'emploi. Bouilloire électrique, Transparent Tools: Manuals, 2012. Design: Jesse Howard. 


\section{Faire exister une relation sensible à la technique}

Aussi, c'est davantage à la notion d'appareil, telle que l'entend le philosophe Pierre-Damien Huyghe, et non à la définition qu'en donne Vilém Flusser en tant que « monstruosité » perfide, que le bricolage par les designers peut renvoyer. Comme il le rappelle : « un appareil est une modalité technique distincte de l'outil et de la machine [...] Quand une technique est employée à titre d'outil, c'est parce qu'elle prolonge, affine, ou augmente une qualité corporelle (Huyghe 2014). Envisager une machine comme un appareil revient alors à la considérer ni comme un automate capable de se passer de l'être humain pour fonctionner, ni uniquement comme un outil en tant que prolongement corporel de ses capacités physiques. Pour lui, un appareil coïncide avec « la capacité à former de la sensibilité » (Huyghe 2014). Si la notion d'appareil est indissociable d'une « puissance de perception » alors on peut certainement étudier les bricolages des designers, notamment les bricolages numériques et la création de machines, selon cette conception. Il y a certes d'une part «sa donne mécanique », c'est-àdire les propriétés mécaniques de l'appareil, sa force motrice, ses composants mécaniques et électroniques, mais il y a aussi les traces qu'il laisse. Dans le cas de la chaise de Dirk Vander Kooij (Endless Pulse Low Chair, 2011), ces « traces » peuvent correspondre à la façon dont la matière est déposée via un bras robotisé qui en définit la course et une filière qui contraint la matière plastique fondue. Dans le champ du design graphique, elles peuvent résulter du processus de codage et de génération des formes. On peut penser à cet égard aux appareils graphiques d'Axel Morales (Piezzographie) notamment celui qui consiste en un procédé d'impression numérique par dépôt d'encres colorées. Assurément, les résultats sont spécifiques et n'auraient pas pu être obtenus autrement. Bricoler des appareils consiste donc, en design, à mettre en place les conditions d'émergence et d'apparition des possibilités esthétiques des programmes et des machines, à « libérer la puissance de l'appareil » (Huyghe 2014), entendue comme potentiel esthétique. Il apparaît que la condition nécessaire au fait de « former de la sensibilité » (Huyghe 2014) dépend de la capacité des designers à être réceptif et à matérialiser des phénomènes. En tant que « travailleurs du sensible (Huyghe 2014), les designers tentent de faire exister une «fabrique du sensible » (Rancière 1999) à partir des machines et des programmes, en mettant au point leurs propres machines et protocoles de production. Le travail du designer consiste alors à « appareiller » les machines, c'est-à-dire à en infléchir les usages afin d'interroger le visible et le tactile, de déplacer les repères et les habitudes de production. Favoriser un bricolage numérique en design est ainsi une façon de faire exister une relation à la technique et aux technologies selon des modalités autres que seulement performatives et fonctionnelles. Ces bricolages numériques ne se rapportent en cela ni à la briccola en tant qu'engin de guerre, ni exactement à des dispositifs fonctionnels performants et industriellement rentables (du moins pas exclusivement). L'enjeu est de taille puisqu'ils concourent à démontrer non seulement que d'autres formes d'industrie sont possibles mais également que la dimension créative à laquelle se rapporte leur activité peut faire exister, au sein du monde contemporain, un rapport sensible aux techniques. 

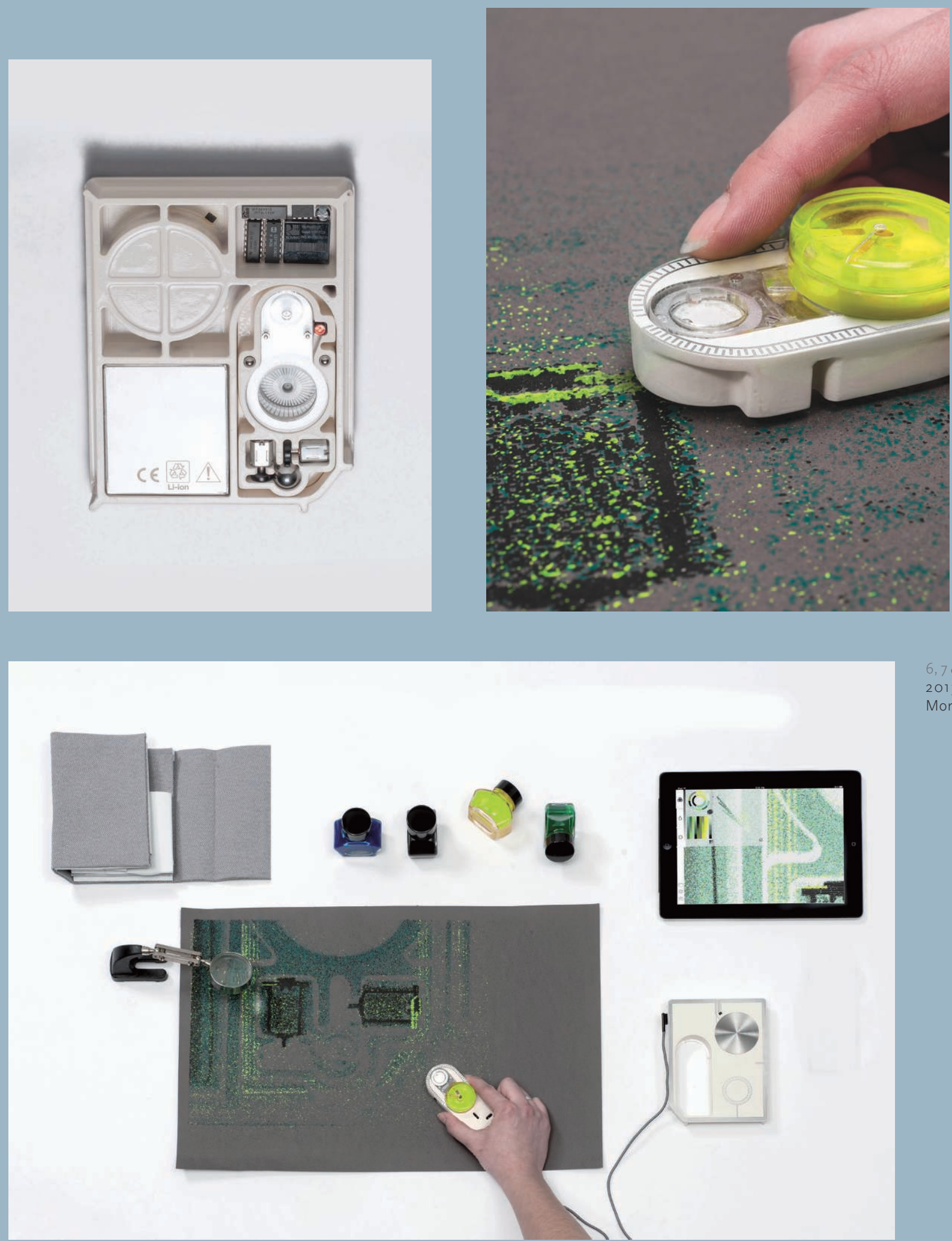

6,7 \& 8. Piezzographie, 2013. Design: Axel

Morales. 


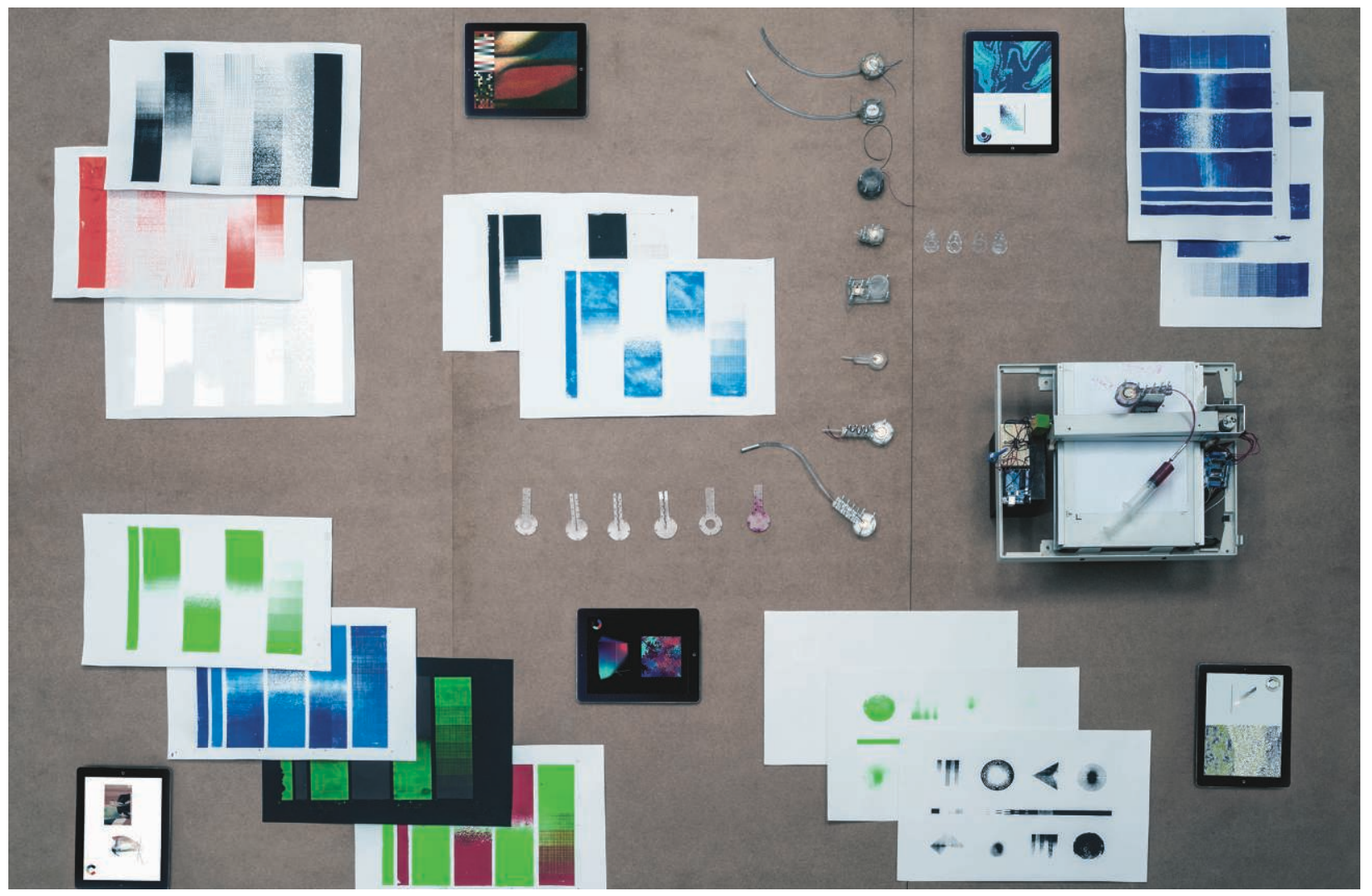

9. Piezzographie, 2013. Design: Axel Morales

\section{Faire avec le numérique}

Ces approches, qui consistent pour les designers à bricoler leurs propres dispositifs productifs, sont intéressantes car elles entretiennent une relation à la technique qui accepte des « résidus d'abstraction » (Simondon 1958: 26). Le caractère artisanal des bricolages qu'ils génèrent (intégrant une part d'improvisation et d'indétermination) n'est ni dommageable, ni à dépasser ; leur « structure floue » (Simondon 1958) n'est pas nécessairement à rendre plus nette, elle est plutôt la condition nécessaire à de possibles interventions, l'occasion pour les designers et les citoyens d'entretenir une plus grande proximité à l'égard des modes de production. En cela, leurs bricolages seraient davantage à situer du côté de la « science du concret » définie par Claude Lévi-Strauss comme caractéristique de la « pensée sauvage » en tant qu'« exploitation spéculative du monde sensible en termes de sensible » (1962) que du côté du processus de concrétisation décrit par Gilbert Simondon en tant que « dépassement de la forme primitive de l'objet technique » et de sa « forme abstraite ». En ce sens, les machines ou appareils qu'ils proposent n'ont pas seulement pour but d'exécuter de façon performante une action en vue d'une fin préétablie, ils sont plutôt une invitation à considérer un rapport à la technique qui ne représente en rien une désillusion ou un renoncement aux technologies numériques actuelles mais bien, au contraire, une façon intelligente 
et sensible de « faire avec » 6 et 7 (de Certeau 1990); à la fois de mieux les connaître mais aussi et surtout de travailler esthétiquement avec elles. Sil serait imprudent de « croire qu'elles [les machines] peuvent tout, parce qu'elles peuvent beaucoup » (Rioux de Maillou 1895), sans doute faut-il surtout attendre des designers qu'ils puissent encore les bricoler sensiblement. Cette relation de réciprocité, entre la machine et ce qu'elle produit, renvoie à une idée moderne qui consiste à mettre en évidence un procédé de fabrication, à exposer sensiblement ses qualités « sui generis » (Rioux de Maillou 1895).

\section{En faveur d'un ubougé " technique}

Les designers, en développant cette attitude propice au bricolage, rencontrent ce que Bruno Latour appelle le « pliage technique » (2010), venant « interrompre, courber, détourner, détourer les autres modes d'existence en introduisant, par une astuce, un différentiel de matériau, de résistance, quel que soit par ailleurs le type de matériau ». Dans le champ du design, cette « astuce » peut correspondre à la façon dont le designer aura bricolé une chose, au script qu'il aura su créer ou modifier pour piloter un programme ou une machine, à la ruse dont il aura su et pu faire preuve pour mettre en forme les matériaux et les convoquer esthétiquement. Ce différentiel, que j’appelle pour ma part un « bougé » (Fétro 2011: 351-353), constitue l'essentiel du travail du designer. C'est cette capacité à prendre des distances avec les usages courants (ce que l'on pourrait appeler des usages réguliers des technologies, c'est-à-dire conformes aux instructions délivrées par les fabricants et exploitants commerciaux), tout en les ayant parfaitement intégrés, qui lui permet de pressentir un résultat sans toutefois exactement le prévoir. C'est à l'aune de ce différentiel, de l'écart qu'il aura su faire exister, du déplacement qu'il aura pu opérer, que son talent pourra être apprécié. Le designer fait œuvre de design à chaque fois qu'il « instaure » ce détour, ce "pliage technique », autrement dit à chaque fois, non pas qu'il se plie à la technique, mais qu'il s'éloigne de ses régulations imposées, déloge la technique de sa droiture, déroge à son efficacité, fait exister un rapport non régulier à la technique. Par « non régulier », il ne faut pas entendre un rapport illégal, exempt de toute règle, mais plutôt une relation non conforme aux habitudes de productions courantes. Les bricolages numériques auxquels ils s'adonnent font exister un différentiel productif qui ne serait pas possible sans eux et qui naurait sans doute pas pu exister autrement. Chacune des démarches qui relèvent du bricolage tel qu'on l'a défini en tant quattitude propice à un « bougé » technique, « célèbre[nt] le sens de la courbe, du biaisé, du déhanché, la marche en crabe de la technique » (Latour 2010). Le designer est donc plus que jamais l'instaurateur d'un résultat surprenant, celui qui met en place les conditions de sa survenue, ni en prévoyant tout à fait ce qui adviendra, ni en se soumettant entièrement aux outils et technologies employés. Car il attend de la technique qu'elle occasionne quelque chose qu'il n’avait pas exactement prévu préalablement, condition même du caractère de nouveauté de ce qu'il pourra générer. Le fait de ne pas se conformer exactement aux règles d'usage et aux programmes de pilotage existants, de fabriquer des machines non conventionnelles permet aux designers de faire exister, à des degrés divers, des ruptures dans les usages et les traditions techniques. Il ne s'agit pas pour le designer d'être dans la stricte observance des règles édictées par un mode d'emploi mais dans un rapport émancipé aux programmes. Sans doute pouvons-nous percevoir là l'un des buts 
essentiels du design qui consiste à ne pas seulement recourir conventionnellement à des techniques mais à les désorienter des habitudes productives. Il cultive, en ce sens, un rapport dédaléen (mais non dramatique) à la technique qui le conduit à faire exister des machines et des bricoles surprenantes parce qu'elles ont su interroger des héritages culturels et des traditions qui, jusque là, semblaient indépassables.

\section{Notes}

1. Dans la Grèce antique, mēkhanē (Bailly 1996 [1895]) désigne aussi bien un engin, une machine de guerre qu'une machine de théâtre permettant de générer la mort, l'effroi mais aussi la surprise (coup de théâtre). Par extension, elle renvoie à une sorte de machination, une invention ingénieuse, une ruse ou un artifice.

2. «RepRap (contraction de l'anglais Replication Rapid Prototyper pouvant se traduire par "concepteur de réplication rapide") est un projet britannique de l'Université de Bath, visant à créer une imprimante tridimensionnelle en grande partie auto-réplicative et libre (c'est-à-dire sans brevet, et dont les plans sont disponibles pour tout le monde) sous licence : Licence publique générale GNU». Wikipédia. [En ligne] URL: fr.wikipedia.org/wiki/ RepRap. Consulté le 11 août 2015.

3. Citons, à titre d'exemple, le Liberator 3D-printed gun de Cody Wilson créé en 2012.

4. Cette notion de repère n'est d'ailleurs pas étrangère à un autre sens du mot «briccola» qui désigne en italien, outre la catapulte, un regroupement de plusieurs poteaux servant d'indicateur maritime aux navigateurs de la lagune de Venise afin d'éviter l'enlisement, signalant ainsi les limites des eaux

navigables. En suivant cette métaphore, le bricolage offensif pourrait correspondre, dans le champ du design, à une limite à ne pas dépasser, du moins à interroger.

5. Ce mot provient de l'espagnol brincar qui signifie faire des cabrioles, sauter, faire de petits bonds.

6. Il ne s'agit pas ici de «faire avec » de façon résignée, en pensant qu'il n'y a pas d'autre choix mais de faire avec les machines numériques, autrement dit, de favoriser un rapprochement avec elles afin d'en explorer les possibilités aussi bien techniques, économiques, sociales, esthétiques qu'écologiques.

7. Michel de Certeau emploie cette formule au chapitre III à propos des usages et des tactiques («Faire avec : usages et tactiques »). On trouve également cette idée dans La Pensée sauvage de Claude Lévi-Strauss lorsqu'il dit : « Le propre de la pensée mythique est de s'exprimer à l'aide d'un répertoire dont la composition est hétéroclite et qui, bien qu'étendu, reste tout de même limité ; pourtant, il faut qu'elle s'en serve, qu'elle que soit la tâche qu'elle s'assigne, car elle n'a rien d'autre sous la main », et de poursuivre quelque lignes suivantes à propos du bricoleur: « son univers instrumental est clos, et la règle de son jeu est de toujours s'arranger avec les "moyens du bord" ».

\section{I'auteure}

Sophie Fétro est maître de conférences, elle enseigne la théorie et la pratique du design à l'Université Paris 1 (PanthéonSorbonne). Ses recherches portent sur les mutations technologiques que connaissent les processus de création en design, en particulier sur les outils numériques d'aide à la conception et à la fabrication.

\section{Iconographie}

Image d'ouverture. Ventilateur, série Hacking Households, 2014, design : Jesse Howard, Leonardo Amico, Thibault-

Brevet, Coralie Gourguechon, Jure Martinec, Nataša Muševič \& Tilen Sepič $\odot$ photographie Tilen Sepič. 
1. Batteur électrique. Série Hacking Households, 2014 Design : Jesse Howard, Leonardo Amico, Thibault Brevet, Coralie Gourguechon, Jure Martinec, Nataša Muševič, and Tilen Sepič $\odot$ photographie Tilen Sepič.

2. Grille pain. Toaster, 2012. Design : Jesse Howard (C) photographie Jesse Howard.

3. Mode d'emploi. Grille pain, Transparent Tools : Manuals, 2012. Design : Jesse Howard @ photographie Jesse Howard.

\section{Références}

Agamben, G. 2007 Qu'est-ce qu'un dispositif? Paris : Payot $\&$ Rivages.

Bailly, A. 1996 [1895] « Mēkhanē » in Dictionnaire grec-français, Paris : Hachette.

Battaglia, S. 1962 Grande dizionario della lingua italiana, II (Balc-Cerr), Turin : Presso la tipografia sociale torinese per conto della Unione Tipografico-Editrice Torinese.

Coulangeon, P., Menger, P.-M. \& Roharik, I. 2002 « Les loisirs des actifs : un reflet de la stratification sociale » (cf. « Nomenclature des activités de loisirs »), Économie et statistique no352-353: 39-55. [En ligne] URL: www. insee.fr/fr/themes/document.asp?reg_id=0\&ref_ id=934. Consulté le 10 août 2015.

Certeau, M. de 1990 L'Invention au quotidien I. Arts de faire, Paris : Gallimard.

Detienne, M. \&Vernant, J.-P. 1974 Les Ruses de l'intelligence. La mètis des Grecs, Paris : Flammarion.

Dupré, P. 1972 Encyclopédie du bon français dans l'usage contemporain, Paris : Éditions de Trévise.

Fétro, S. 2012 « Marc Fornes : Double Agent White », Strabic [revue en ligne]. [En ligne] URL : strabic.fr/DoubleAgent-White-prototype-d. Consulté le 10 août 2015.

- 2011 Étude critique du merveilleux en design : tours et détours dans les pratiques d'assistance au projet, thèse de doctorat, Université Paris I.

Flusser, V. 1996 Choses et non-choses - Esquisses phénoménologiques, Nîmes : Jacqueline Chambon.

Furetière, A., Bayle, P. (préface) \& Rey, A. (introduction) 1978 [1690] Le Dictionnaire Universel d'Antoine Furetière, Paris : SNL/Le Robert.

Gimpel, J. 2002 [1975] La Révolution industrielle du Moyen Âge, Paris : Seuil.

\section{Pour citer cet article}

Fétro, S. 2015 « Bricolages en design. Inventer des rapports non réguliers à la technique », TechniquesECulture nº 64 «Essais de Bricologie. Ethnologie de l’art et du design contemporains », p. 152-167.
4. Bouilloire électrique. Electric Kettle, 2012. Design : Jesse Howard @ photographie Jesse Howard.

5. Mode d'emploi. Bouilloire électrique, Transpa(C) photographie Jesse Howard.

6, 7, 8 \& 9. Piezzographie, 2013. Design : Axel Morales (c) photographie Véronique Huyghe.

Huyghe, P.-D. 2003 «Introduction » du dossier « Temps et appareils $»$, [Plastik] n ${ }^{\circ} 3$.

Imbs, P. (dir.) 1975 « Bricole » in Trésor de la langue française, dictionnaire de la langue du Xixe et du Xxe s. (1789-1960), tome 4, Paris : Centre National de la Recherche Scientifique - Nancy.

Latour, B. 2010 « Prendre le pli des techniques », numéro spécial de la revue Réseaux n¹63, vol. 28.

Lévi-Strauss, C. 1962 La Pensée sauvage, Paris : Plon.

Littré, É. 2000 [1873-1877] Le Littré dictionnaire de la langue française en un volume, Paris : Hachette.

Moholy-Nagy, L. 1993 [1947] « Nouvelle méthode d'approche. Le design pour la vie », Peinture, Photographie, Film et autres écrits sur la photographie, Nîmes : Jacqueline Chambon.

Panckoucke, A. J. 1750 Dictionnaire des proverbes françois, et des façons de parler comiques, burlesques et familiers, avec l'explication, et les étymologies les plus avérées, Francfort et Mayence: F. Varrentrapp.

Rancière, J. 2000 Le Partage du sensible, Paris : La Fabrique. Rey, A. (dir.) 2005 Dictionnaire culturel en langue française, Paris : Dictionnaire Le Robert - SEJER.

Rioux de Maillou, P. 1895 «Les Arts décoratifs et les machines, $1895 »$, Revue des arts décoratifs XV : 267-273 in N. McWilliam, C. Méneux et J. Ramos (dir.) 2014 L’Art social de la Révolution à la Grande Guerre. Anthologie de textes sources, Paris : INHA (« Sources »). [En ligne] URL: inha.revues.org/5762. Consulté le 31 juillet 2015.

Simondon, G. 1989 [1958] Du mode d'existence des objets techniques, Paris : Aubier. rent Tools: Manuals, 2012. Design : Jesse Howard 